\title{
In vitro digestion of lactoferrin-glycomacropeptide nanohydrogels incorporating bioactive compounds: Effect of a chitosan coating
}

\author{
Ana I. Bourbon ${ }^{\mathrm{a}, \mathrm{b}, *}$, Ana C. Pinheiro ${ }^{\mathrm{a}, \mathrm{c}}$, Miguel A. Cerqueira ${ }^{\mathrm{a}, \mathrm{b}}$, António A. Vicente ${ }^{\mathrm{a}}$ \\ ${ }^{\text {a }}$ CEB, Centre of Biological Engineering, University of Minho, Campus de Gualtar, 4710-057 Braga, Portugal

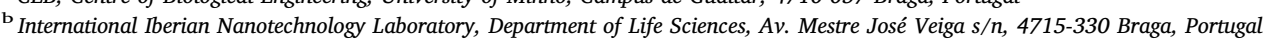 \\ ${ }^{\mathrm{c}}$ Instituto de Biologia Experimental e Tecnológica, Avenida da República, Quinta-do- Marquês, Estação Agronómica Nacional, Apartado 12, 2781-901 Oeiras, Portugal
}

\section{A R T I C L E I N F O}

\section{Keywords:}

Nanotechnology

Functional compounds

Curcumin

Caffeine

\begin{abstract}
A B S T R A C T
The behaviour of lactoferrin (Lf) - glycomacropeptide (GMP) nanohydrogels with and without a chitosan coating was evaluated during gastrointestinal digestion. The application of a chitosan coating allowed to reduce the protein degradation from $86 \%$ to $76 \%$ for Lf and from $71 \%$ to $53 \%$ for GMP.

Protein bioaccessibility results showed that in nanohydrogels with chitosan coating $23 \%$ of Lf and $40 \%$ of GMP remained intact until absorption. Based on these results, the bioaccessibility of two different bioactive compounds encapsulated in Lf-GMP nanohydrogels with chitosan coating was evaluated during gastrointestinal digestion. Curcumin was used as lipophilic model compound and caffeine as a hydrophilic model compound. Bioaccessibility of curcumin in coated nanohydrogels was $72 \%$ while the corresponding value for curcumin in free form only reached $66 \%$. It was also observed that under simulated gastric and intestinal conditions, free curcumin lost around $68 \%$ of its antioxidant activity while when incorporated into nanohydrogels only $30 \%$ of this activity was lost. Results also showed that the bioaccessibility of caffeine encapsulated in coated nanohydrogels was $63 \%$ while caffeine in free form only reached $59 \%$.
\end{abstract}

\section{Introduction}

The growing awareness of the relevance of food in health maintenance and the difficulties of changing eating habits, calls for an enrichment of foods with bioactive compounds. One of the challenges of food enrichment with such bioactive compounds is related to the poor solubility, in the case of hydrophobic compounds, in food matrices and their instability during digestion and consequent poor bioavailability. These challenges are promoting research efforts to find more effective delivery systems based on natural biopolymers (Livney, 2012). Protein nanohydrogels are promising systems to be used as carriers of bioactive compounds in food products (Martins et al., 2015). The high nutritional value, non-toxicity and ability of proteins to bind to hydrophobic and hydrophilic bioactive compounds make them a useful vehicle to help to incorporate such bioactives in foods (Wong, Camirand, Pavlath, Parris, \& Friedman, 1996). However, during the gastric digestion step, proteins are denatured by environmental conditions (low $\mathrm{pH}$ and high ionic force) and hydrolyzed by enzymes (pepsin) (Yvon, Beucher, Scanff, Thirouin, \& Pelissier, 1992). One of the strategies to improve the protein nanohydrogels stability during enzymatic digestion and the controlled release of active ingredients during gastric and intestinal digestion is the addition of a polysaccharide coating (Liu, Gao, \& Yuan, 2014).

In our previous work, nanohydrogels composed by lactoferrin (Lf) and glycomacropeptide (GMP) were coated with a chitosan layer (Bourbon, Pinheiro, Cerqueira, \& Vicente, 2016) and the stability of protein nanohydrogels with chitosan coating and the release properties of caffeine were studied during gastric digestion. The addition of a chitosan coating on Lf-GMP nanohydrogels had a positive effect on the stability of Lf-GMP nanohydrogels during gastric digestion, once the presence of nanohydrogels coated with chitosan was stable for longer periods of time. During the gastric digestion, the hydrolysis of proteins was slower in nanohydrogels coated with chitosan than in nanohydrogels alone. It was also observed that the release of caffeine was governed by Fick's diffusion and by relaxation of the matrix, for nanohydrogels with and without chitosan coating. However in nanohydrogels with the presence of a chitosan coating, the mass of caffeine released was lower, showing that the chitosan coating can be used to control the initial "burst release" of nanohydrogels without coating. Despite all these findings, more information is needed to understand the protein-based nanostructures digestion process and its impact on the release and uptake of encapsulated bioactive compounds under specific

\footnotetext{
* Corresponding author. International Iberian Nanotechnology Laboratory, Department of Life Sciences, Av. Mestre José Veiga s/n, 4715-330 Braga, Portugal.

E-mail address: isabelbourbon@gmail.com (A.I. Bourbon).
} 
gastrointestinal conditions. In vitro digestion models have recently gained much attention as a tool for understanding the basic physiochemical processes that occur during the digestion and the release of encapsulated compounds (Ahmed, Li, McClements, \& Xiao, 2012). To exert a health benefit, bioactive compounds need to withstand food processing, be released from the food matrix after ingestion and be bioaccessible in the gastrointestinal tract, undergo metabolism and reach the target tissue of action (Rein et al., 2013). Due to the complexity of gastric and intestinal digestion and the many factors affecting their transition during digestion (e.g. pH, ionic strength, enzymes), unravelling the factors affecting the bioaccessibility of bioactive compounds is still a challenge. In fact, the assessment of the bioaccessibility of a health bioactive compounds is important for the understanding of the relationship between food and nutrition (Rein et al., 2013).

In the present study, a dynamic in vitro digestion model was used to evaluate the stability and bioaccessibility of protein-based nanohydrogels coated with a chitosan layer and to evaluate the bioaccessibility of two bioactive compounds when loaded in these coated nanohydrogels. Curcumin, as a lipophilic model compound and caffeine as a hydrophilic model compound were encapsulated in Lf-GMP nanohydrogels coated with a chitosan layer and their bioaccessibility, was evaluated during in vitro digestion and compared with free solutions of curcumin and caffeine. The digestion process was carried out in a dynamic model that mimics the environmental (temperature, $\mathrm{pH}$, ionic strength) and physical conditions (peristaltic and intestinal movements) of the gastrointestinal tract. The knowledge obtained from this study will be relevant to better design our future foods, containing new delivery systems for enhancing the bioaccessibility of health-promoting compounds.

\section{Materials and methods}

\subsection{Materials}

Purified lactoferrin (Lf) powder was obtained from DMV International (USA) and it is composed by $96 \%$ protein, $0.5 \%$ ash, $3.5 \%$ moisture and an iron content is around $120 \mathrm{ppm}$ (composition expressed as a dry weight percentage). Commercial glycomacropeptide (GMP) was kindly offered by Davisco Food International, INC. (Le Sueur, USA) and its reported composition is: $82.5 \%$ protein, $1 \%$ fat, $7 \%$ ash and 7\% moisture. Chitosan of low molecular weight (molecular weight ranging between 50 and $100 \mathrm{kDa}$ and with a deacetylation degree $\geq 95 \%$ ) was purchased from Sigma-Aldrich (St. Louis, MO, USA). Lactic acid (90\%) was obtained from Acros Organics (Geel, Belgium). Curcumin was purchased from Sigma-Aldrich (St. Louis) and caffeine was purchased from VWR (USA). All the samples were dissolved in deionized water purified to a resistance of $15 \mathrm{M} \Omega$ (Millipore, France).

\subsection{Encapsulation of bioactive compounds in Lf-GMP nanohydrogels coated with chitosan layer}

Lf-GMP nanohydrogels were prepared as described in Bourbon et al. (2016). Briefly, $0.02 \%(w / w)$ of Lf and $0.02 \%(w / w)$ of GMP were dissolved separately, in deionized water purified at $25^{\circ} \mathrm{C}$. The $\mathrm{pH}$ values of biopolymer solutions were separately adjusted to 5.0, with $0.1 \mathrm{~mol} . \mathrm{L}^{-1}$ of hydrochloric acid. Lf aqueous solution was added dropwise into GMP aqueous solution with gentle stirring until the final molar ratio of volume (MR) 1:7 of Lf to GMP. At this stage, bioactive compounds were added to the mixture.

A maximum encapsulation efficiency of $95.12 \pm 1.4$ and $90.02 \pm 2.1 \%$ was obtained when $0.082 \mathrm{mg} \mathrm{mL}^{-1}$ of curcumin and $0.03 \mathrm{mg} \mathrm{mL}^{-1}$ of caffeine was encapsulated in Lf-GMP nanohydrogels (Sanguansri \& Augustin, 2006). Briefly, curcumin solution $\left(0.082 \mathrm{mg} \mathrm{mL}^{-1}\right.$ ) or caffeine solution $\left(0.03 \mathrm{mg} \mathrm{mL}^{-1}\right)$ was added to the mixture solution of $\mathrm{Lf}$ $(0.02 \%(\mathrm{w} / \mathrm{w}), \mathrm{pH} 5.0)$ and GMP $(0.02 \%(\mathrm{w} / \mathrm{w}), \mathrm{pH} 5.0)$ with a molar ratio of $1: 7$ of Lf to GMP at $25^{\circ} \mathrm{C}$. After gentle stirring for $30 \mathrm{~min}$, the mixture of
Lf-GMP with bioactive compounds was subsequently heated at $80^{\circ} \mathrm{C}$ for $20 \mathrm{~min}$ in a water bath (closed system) to obtain a homogeneously dispersed nanohydrogel solution. The chitosan was assembled on the Lf-GMP nanohydrogels by the layer-by-layer (LbL) deposition technique. Nanohydrogels with bioactive compounds encapsulated were added to a chitosan solution ( $1 \mathrm{mg} \mathrm{mL}^{-1}, \mathrm{pH} 3$, dissolved in $1 \%$ of lactic acid) at volume ratios (VR) of 0.1 of Lf-GMP nanohydrogels to chitosan, with constant stirring of $200 \mathrm{rpm}$ during $15 \mathrm{~min}$, creating the nanohydrogels with a coating (Bourbon et al., 2016).

\subsection{In vitro digestion}

\subsubsection{Gastrointestinal model}

A dynamic gastrointestinal model system was used in the in vitro digestion experiments. This model simulates the main events that occur during digestion and consists of four compartments simulating the stomach, duodenum, jejunum and ileum. Each compartment consists in two connected glass reactors with a flexible wall inside and water is pumped around the flexible walls to maintain the temperature at $37^{\circ} \mathrm{C}$ and to enable the simulation of the peristaltic movements (by the alternate compression and relaxation of the flexible walls). The changes in water pressure are achieved by peristaltic pumps which alter the flow direction according to the time-controlling devices connected to them. The compartments are connected by silicone tubes and, at a predefined time, a constant volume of chyme is transferred. All compartments are equipped with $\mathrm{pH}$ electrodes and $\mathrm{pH}$ values are controlled by the secretion of $\mathrm{HCl}\left(1 \mathrm{~mol} . \mathrm{L}^{-1}\right)$ into the stomach and $\mathrm{NaHCO}_{3}\left(1 \mathrm{~mol} . \mathrm{L}^{-1}\right)$ into the intestinal compartments. The gastric and intestinal secretions are added via syringe pumps at pre-set flow rates. The jejunum and ileum compartments are connected with hollow-fibre devices (SpectrumLabs Minikros, M20S-100-01P, USA) to absorb digestion products and water from the chyme and to modify electrolyte and bile salts concentration of the chyme (Reis et al., 2008).

\subsubsection{Experimental conditions}

In vitro digestion was performed as described by other authors (Reis et al., 2008) with some modifications. A volume of $60 \mathrm{~mL}$ of nanohydrogels with chitosan coating (with and without bioactive compounds encapsulated) was introduced into the dynamic gastrointestinal system (gastric compartment) and the experiment was run for a total of $5 \mathrm{~h}$, simulating average physiological conditions of GI tract by the continuous addition of gastric, duodenum, jejunal and ileal secretions. The gastric secretion consisted of pepsin and lipase in a gastric electrolyte solution ( $\mathrm{NaCl} 4.8$ g.L $\mathrm{L}^{-1}, \mathrm{KCl} 2.2 \mathrm{~g} . \mathrm{L}^{-1}, \mathrm{CaCl}_{2} 0.22$ g.L $\mathrm{L}^{-1}$ and $\mathrm{NaHCO}_{3}$ 1.5 g.L $\mathrm{L}^{-1}$ ), secreted at a flow rate of $0.33 \mathrm{~mL} \mathrm{~min}^{-1}$. The $\mathrm{pH}$ was controlled to follow a predetermined curve (from 4.8 at $t=0$ to 1.7 at $t=120 \mathrm{~min})$ by secreting $\mathrm{HCl}\left(1 \mathrm{~mol} . \mathrm{L}^{-1}\right)$.

The duodenal secretion consisted of a mixture of $4 \%(\mathrm{w} / \mathrm{v})$ porcine bile extract, $7 \%(\mathrm{w} / \mathrm{v})$ pancreatin solution and small intestinal electrolyte solution (SIES) ( $\mathrm{NaCl} 5$ g.L $\mathrm{L}^{-1}, \mathrm{KCl} 0.6$ g.L $\mathrm{L}^{-1}, \mathrm{CaCl}_{2} 0.25$ g.L ${ }^{-1}$ ) secreted at a flow rate of $0.66 \mathrm{~mL} \mathrm{~min}^{-1}$. The jejunal secretion fluid consisted of SIES containing $10 \%(\mathrm{v} / \mathrm{v})$ porcine bile extract solution at a flow rate of $2.13 \mathrm{~mL} \mathrm{~min}^{-1}$. The ileal secretion fluid consisted of SIES at a flow rate of $2.0 \mathrm{~mL} \mathrm{~min}^{-1}$. The $\mathrm{pH}$ of the different parts of small intestine was controlled by the addition of $1 \mathrm{~mol}^{-\mathrm{L}^{-1}} \mathrm{NaHCO}_{3}$ solution to set-points of 6.5, 6.8 and 7.2 for simulated duodenum, jejunum and ileum, respectively. During in vitro digestion, samples were collected directly from the lumen of the different compartments, from the jejunal and ileal filtrates and from the ileal delivery. The jejunal and ileal filtrates were used to determine the bioavailable fraction of bioactive compounds. The samples were tested in the dynamic gastrointestinal model at least in triplicate.

\subsection{Size distribution}

Nanohydrogels with and without chitosan coating were characterized in 
terms of size distribution (by intensity) using a Dynamic Light Scattering (DLS) apparatus (Zetasizer Nano ZS, Malvern Instruments, UK) during their passage through the gastrointestinal system. A sample from each compartment (stomach, duodenum, jejunal filtrate, ileal filtrate and ileal delivery) was analyzed. All measurements were performed at $25^{\circ} \mathrm{C}$. The results are given as the average \pm standard deviation of nine measurements.

The size distribution was evaluated based on the speed at which the particles are diffusing due to Brownian motion. This is done by measuring the rate at which the intensity of the scattered light fluctuates when detected using a suitable optical arrangement. Particle size is calculated based on the Stokes-Einstein equation (Equation (1)):

$d(H)=\frac{K T}{3 \pi D}$

Where $d(H)$ is the hydrodynamic diameter of particles, $K$ is the Boltzmann constant, $T$ is the temperature in Kelvin (K), $D$ is the translational diffusion coefficient and $\eta$ is the medium viscosity (Su \& Zhong, 2016).

\subsection{Quantitative analysis of $L f$ and GMP}

The concentration of Lf and GMP during gastrointestinal digestion was evaluated using high-performance liquid chromatography (HPLC). Protein concentrations were assayed by reversed-phase HPLC on an ACE $5 \mathrm{C}_{18}$ column $(250 \times 4.6 \mathrm{~mm}, 5 \mu \mathrm{m}, 300 \AA$, Advanced Chromatography Technologies, Scotland) with an ACE $5 \mathrm{C}_{18}$ guard cartridge (Advanced Chromatography Technologies, Scotland) at room temperature. The RP-HPLC analysis was performed using an Agilent 1220 HPLC system (Agilent Technologies, Germany). Data acquisition was performed through the Agilent ChemStation software (revision A.10.02) (Agilent Technologies, Palo Alto, CA, USA) and absorbance was measured at the wavelength of $220 \mathrm{~nm}$. A constant flow rate of $0.7 \mathrm{~mL} \mathrm{~min}^{-1}$ was used and the injection volume was $20 \mu \mathrm{L}$. Regarding solvents, solvent A was $0.1 \%(\mathrm{v} / \mathrm{v})$ trifluoroacetic (TFA) in Milli-Q water, and solvent B was $0.1 \%(\mathrm{v} / \mathrm{v})$ TFA in $100 \%(\mathrm{v} / \mathrm{v})$ acetonitrile.

Elution was performed as follows: $100 \%$ A for 5 min, linear gradient of $0-50 \%$ B for $50 \mathrm{~min}$, from 50 to $100 \%$ B over the next $2.5 \mathrm{~min}$ and then maintained at $100 \%$ B for $2.5 \mathrm{~min}$. Finally, solvent A was increased to $100 \%$ in $5 \mathrm{~min}$ and the column was re-equilibrated for $5 \mathrm{~min}$ more.

\subsection{Transmission electron microscopy}

The digestion process was monitored using transmission electron microscopy (TEM). TEM micrographs were conducted on a Zeiss EM 902A (Thornwood, N.Y., U.S.A.) microscope at accelerating voltages of $50 \mathrm{kV}$ and $80 \mathrm{kV}$. The samples were prepared by dropping the solutions onto copper grids coated with carbon film and followed by natural drying.

\subsection{Quantitative analysis of curcumin and caffeine}

Curcumin was determined using an HPLC apparatus comprising a Varian 9002 pump, a Marathon Basic autosampler with a $50 \mu \mathrm{L}$ loop, a Jasco FP-920 Fluorescence detector $\left(\lambda_{\text {ex }}=420 \mathrm{~nm} ; \lambda_{\text {em }}=540 \mathrm{~nm}\right)$ and a Galaxie chromatography data system. The analytical column was a $\mathrm{C}_{18}$ reversed phase YMC-Pack ODS-AQ $(250 \times 4.6 \mathrm{~mm} ; 5 \mu \mathrm{m})$ fitted with a pre-column of the same stationary phase. The mobile phase was a mixture of acetonitrile/acetic acid ( $2 \%(\mathrm{v} / \mathrm{v}))(53: 47)$ that was filtered and degassed with a $0.2 \mu \mathrm{m}$ membrane filter. The flow rate was set to $1 \mathrm{~mL} \mathrm{~min}^{-1}$, and the column temperature was $25^{\circ} \mathrm{C}$.

The identification and quantification of caffeine were also performed by HPLC, with $\mathrm{a}_{18}$ reversed phase $(250 \times 4.6 \mathrm{~mm} ; 5 \mu \mathrm{m})$. The mobile phase consisted of acetonitrile and water $(20: 80 \mathrm{v} / \mathrm{v})$ at a flow rate of $1 \mathrm{~mL} \mathrm{~min}^{-1}$, and the column temperature was $25^{\circ} \mathrm{C}$. Caffeine was detected using a UV detector set at $276 \mathrm{~nm}$. A standard curve with pure caffeine was used to determine the concentration of caffeine compound.
All the samples from gastric and intestinal digestion were subjected to a centrifugation at $10000 \mathrm{~g}$ during $5 \mathrm{~min}$ and a filtration through a $0.45 \mu \mathrm{m}$ membrane. After this, the collected samples were analyzed in HPLC.

\subsection{Antioxidant activity of curcumin}

The free-radical scavenging of curcumin was analyzed using 1,1diphenyl-2-picrylhydrazyl (DPPH) test according to the method of Blois (1958), with some modifications. BHT and BHA were used as positive controls. Briefly, $0.2 \mathrm{~mL}$ of ethanol and $0.3 \mathrm{~mL}$ of the sample dissolved in ethanol (concentrations ranging from 0.025 to $1.0 \mathrm{mg} \mathrm{mL}^{-1}$ ) were mixed in a $10 \mathrm{~mL}$ test tube. DPPH $\left(2.5 \mathrm{~mL}\right.$ of $75 \mu \mathrm{mol} . \mathrm{L}^{-1}$ in ethanol) were then added to achieve a final volume of $3.0 \mathrm{~mL}$ The solution was kept at room temperature for $30 \mathrm{~min}$ and the absorbance was measured at $517 \mathrm{~nm}$ (Blois, 1958):

DPPH Radical Scavenging $(\%)=\frac{A_{0}-\left(A-A_{b}\right)}{A_{0}} \times 100$

where $A_{O}$ is the absorbance at $517 \mathrm{~nm}$ of DPPH without a sample, $A$ is the absorbance of the sample and DPPH at $517 \mathrm{~nm}$ and $A_{b}$ is the absorbance of the sample without DPPH at $517 \mathrm{~nm}$. The absorbance measurements were performed in Elisa Biotech Synergy HT (Biotek, USA).

In order to evaluate the antioxidant effect of curcumin without the interference of Lf-GMP nanohydrogel with chitosan coating and without the interference of gastric and intestinal solution, the effects of these solutions were subtracted from samples.

\subsection{Statistical analyses}

The statistical analyses of the data were performed using Analysis of Variance (ANOVA), Tukey means comparison test $(p<0.05)$ and regression analysis (EXCEL 2007 and SigmaStat, trial version, 2003, USA).

\section{Results and discussion}

\subsection{Effect of chitosan coating on bioaccessibility of proteins}

One of the challenges of using protein structures in food products is their behaviour during the gastrointestinal digestion. Milk proteins have been reported to have numerous functional properties, however ensuring that their structure, form and activity remain intact until they reach the delivery target is still a concern for food developers and the food industry in general. We have observed in a previous work that a chitosan coating improves the stability of Lf-GMP nanohydrogels and decreases the proteins' hydrolysis during gastrointestinal digestion (Bourbon et al., 2016). In order to evaluate the effect of chitosan coating on bioaccessibility of proteins, a gastrointestinal digestion was performed in an in vitro dynamic system. Distribution of protein available (Lf and GMP) in the different compartments of the dynamic gastrointestinal system is presented in Fig. 1.

Fig. 1A shows that the Lf digested was significantly $(p<0.05)$ higher in nanohydrogels without chitosan coating. At the end of $5 \mathrm{~h}$ of simulated gastrointestinal digestion, $76.0 \pm 2.1 \%$ of Lf present in coated nanohydrogels was hydrolyzed while in nanohydrogels without coating $86.1 \pm 3.2 \%$ of Lf has been hydrolyzed. This result revealed that $24.2 \pm 2.1 \%$ of $\mathrm{Lf}$ in coated nanohydrogels was intact at the end of gastrointestinal digestion. Moreover, $18.1 \pm 1.2 \%$ was recovered in the jejunal filtrate, suggesting that this part of small intestine is the main site of absorption of the intact Lf, whereas $5.2 \pm 0.8 \%$ of Lf was recovered in the ileal filtrate. Comparing nanohydrogels with and without chitosan coating, it is possible to observe a difference of less than $5 \%$ of intact Lf absorbed in the ileal filtrate (Fig. 1A). It is during the gastric digestion (stomach compartment) that a higher amount of Lf 

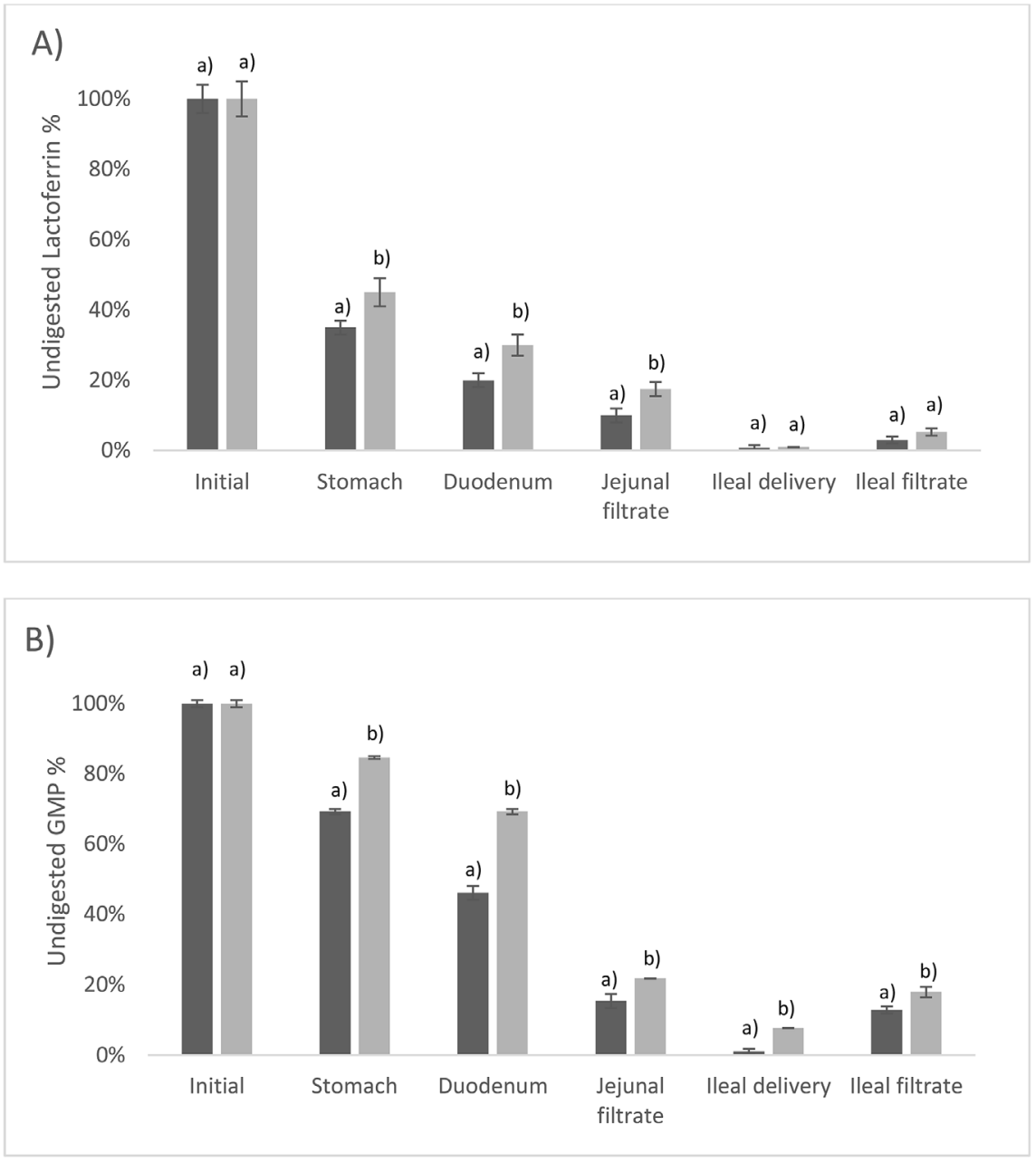

Fig. 1. - Undigested percentage of Lf (A) and GMP (B) in the different compartments of the dynamic gastrointestinal system during digestion of nanohydrogels ( $\boldsymbol{\square})$ and nanohydrogels coated with a chitosan layer ( $\square$ ) (data are presented as mean $\pm 95 \%$ confidence interval; values followed by different superscript letters are significantly different $(p<0.05))$. is digested: $45.1 \pm 0.4 \%$ and $35.2 \pm 1.2 \%$ for nanohydrogels without and with chitosan coating, respectively. According to Yvon et al. (1992), it is in the stomach that almost all the protein is hydrolyzed by enzymatic effect (pepsin), being the low $\mathrm{pH}$ values and high ionic force parameters that have a high impact in denaturation of proteins during the gastric digestion (Yvon et al., 1992). Different authors observed that almost $60 \%$ of $\mathrm{Lf}$ is digested during gastrointestinal tract, majority after the passage through the stomach (Bokkhim, Bansal, Grøndahl, \& Bhandari, 2016; Troost, Steijns, Saris, \& Brummer, 2001). Also, Inglingstad et al. (2010) observed that Lf was highly degraded by human gastrointestinal enzymes during its passage through the gastrointestinal tract (Inglingstad et al., 2010).

Results showed that $29.1 \pm 1.2 \%$ and $47.2 \pm 0.9 \%$ of GMP was intact at the end of digestion in nanohydrogels without and with chitosan coating, respectively (Fig. 1B). Whereby, $15.3 \pm 0.2 \%$ and $22.1 \pm 0.4 \%$ of intact GMP was present in the jejunal filtrate and $13.1 \pm 0.1 \%$ and $18.2 \pm 0.1 \%$ was present in the ileal filtrate for nanohydrogels without and with chitosan coating, respectively. Comparing with Lf is possible to observe that GMP is more resistant to gastrointestinal conditions. These results are in concordance with findings obtained by other authors, which reported that GMP was more resistant to pepsin than other milk proteins (e.g. immunoglobulin $\mathrm{G}$ and $\beta$-lactoglobulin) (Bokkhim et al., 2016; Inglingstad et al., 2010; Yvon et al., 1992). Nabil, Gauthier, Drouin, Poubelle, and Pouliot (2011) observed that during the digestion of bovine whey protein extracts, GMP was one of the proteins undigested at the end of $1 \mathrm{~h}$ of gastric digestion (Nabil et al., 2011).

Fig. 1 also shows that the application of the chitosan coating on protein-based nanohydrogels led to higher intact amounts of Lf and
GMP during the gastrointestinal digestion, which was thus available to be absorbed in jejunum and ileum compartments. In a previous work, it was observed that the chemical interactions established between chitosan and nanohydrogels led to a decrease of protein (Lf and GMP) degradation during gastric digestion. Moreover, it was observed that the presence of chitosan coating improved the stability of nanohydrogels for longer periods during gastric digestion. This can be due to the fact that chitosan in contact with gastric juice forms a hydrogel, protecting the protein system (Anal, Bhopatkar, Tokura, Tamura, \& Stevens, 2003). In intestinal digestion conditions, chitosan can be used to retard the adsorption of bile salts and/or lipase, restricting the access of the enzyme to the substrate and reducing the ability of the system to solubilize digestion products (Liu et al., 2014).

Particle size distribution and TEM images of nanohydrogels with and without chitosan coating in different compartments of the dynamic gastrointestinal system during digestion are presented in Fig. 2 and Fig. 3, respectively.

Fig. 2 shows that nanohydrogels coated with chitosan layer (Fig. 2A) present a single peak still in the stomach compartment, suggesting that nanohydrogels are stable in solution and do not present particle aggregation. In a previous work, it was observed by native electrophoresis that Lf-GMP nanohydrogels with a chitosan coating are intact until $90 \mathrm{~min}$ in a gastric solution (Bourbon et al., 2016). Fig. 3A, in the stomach compartment, shows that nanohydrogels coated with chitosan did not display significant morphological differences compared with the initial sample, which is in concordance with the size distribution. Observing the digestion of nanohydrogels without chitosan coating (Figs. 2B and 3B) it is possible to verify that these nanohydrogels are more unstable. The presence of a bimodal particle size 
A

Initial
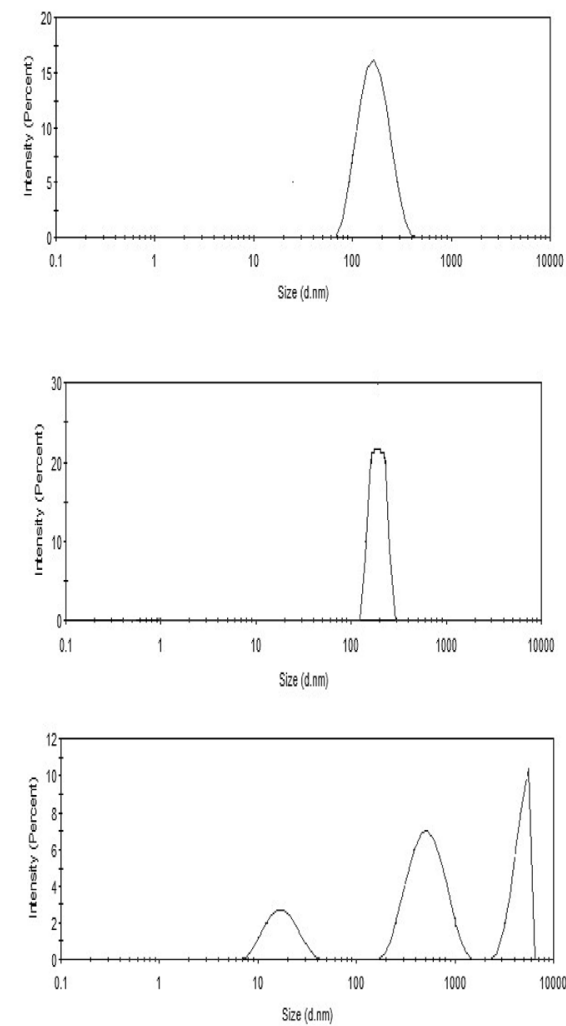

Jejunal

filtrate

Duodenum

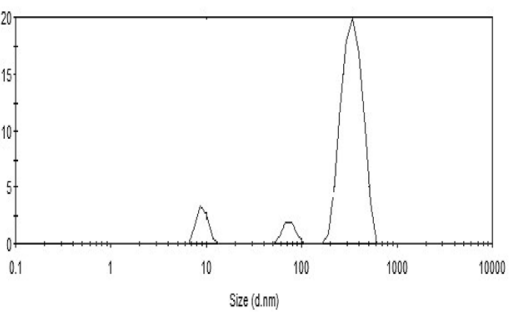

Ileal delivery

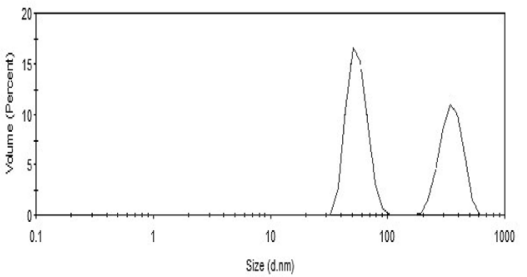

Ileal filtrate
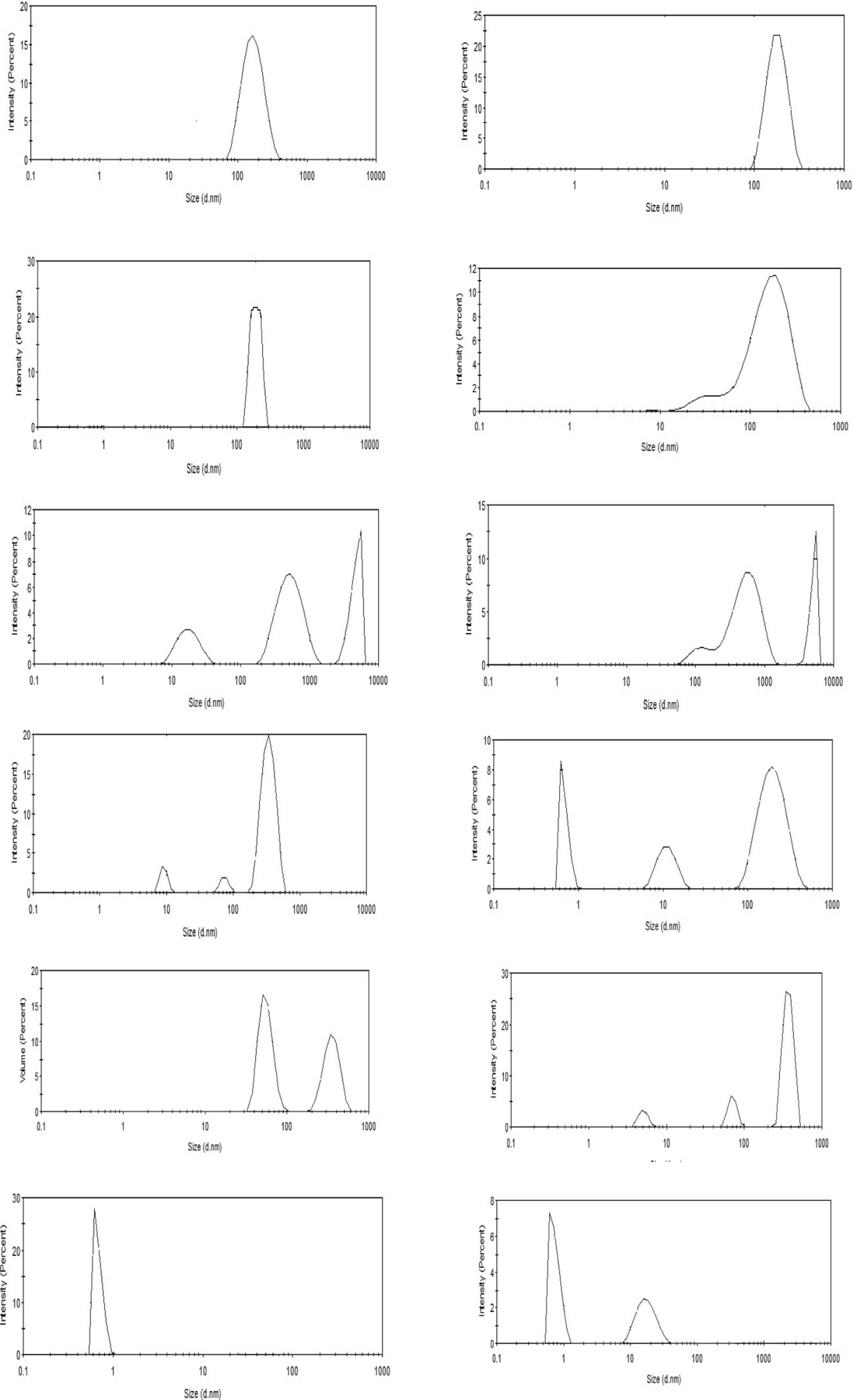

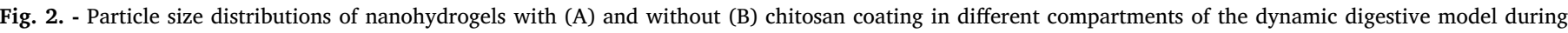
gastrointestinal digestion.

distribution (Fig. 2B) in the stomach compartment suggests that in this stage protein nanohydrogels are being degraded faster than in nanohydrogels with chitosan coating. It is also possible to observe that in this stage, proteins agglomerates are formed.
In the duodenum, the size distributions of nanohydrogels coated and uncoated with chitosan layer present three peaks, indicating the presence of small and large particles in solution (Fig. 3A and B). In fact, for the duodenum compartment shows the presence of larger particles 
A

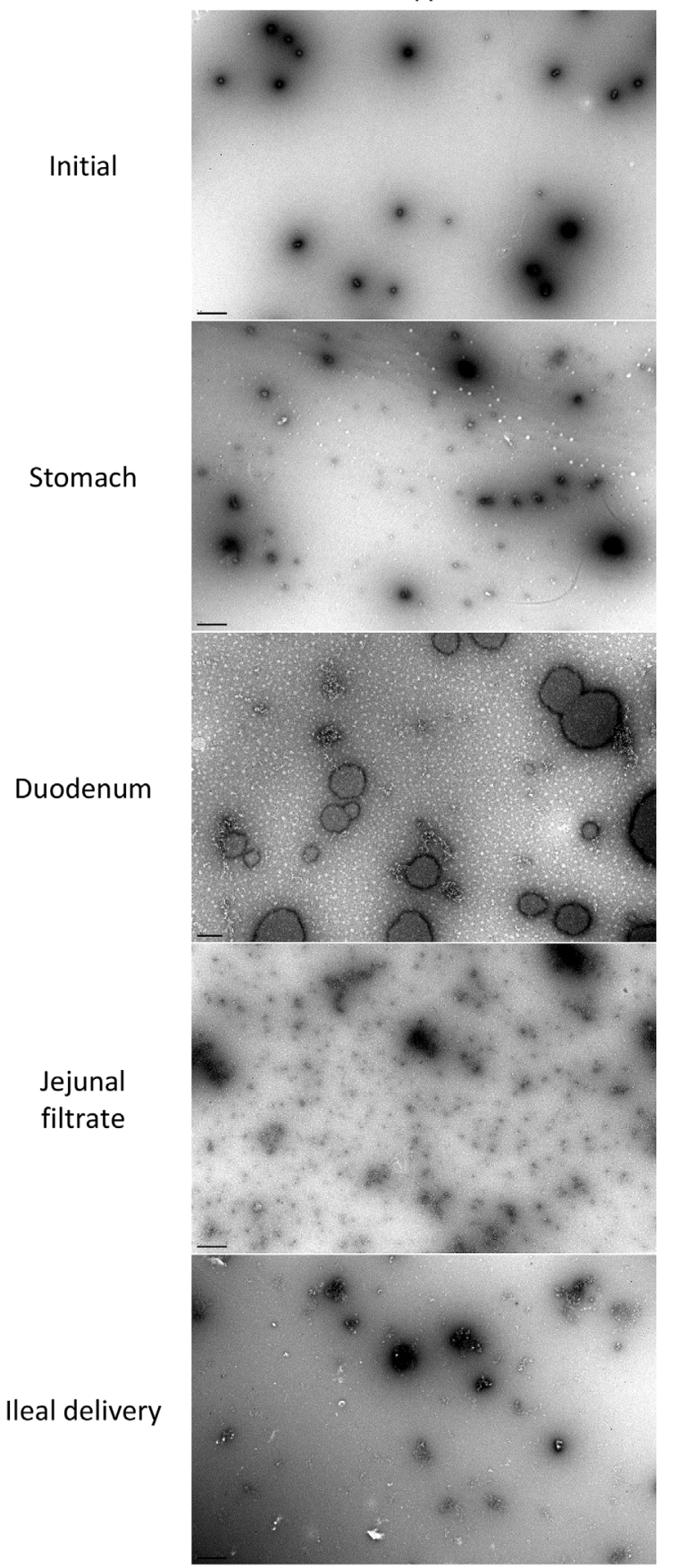

$\mathrm{B}$

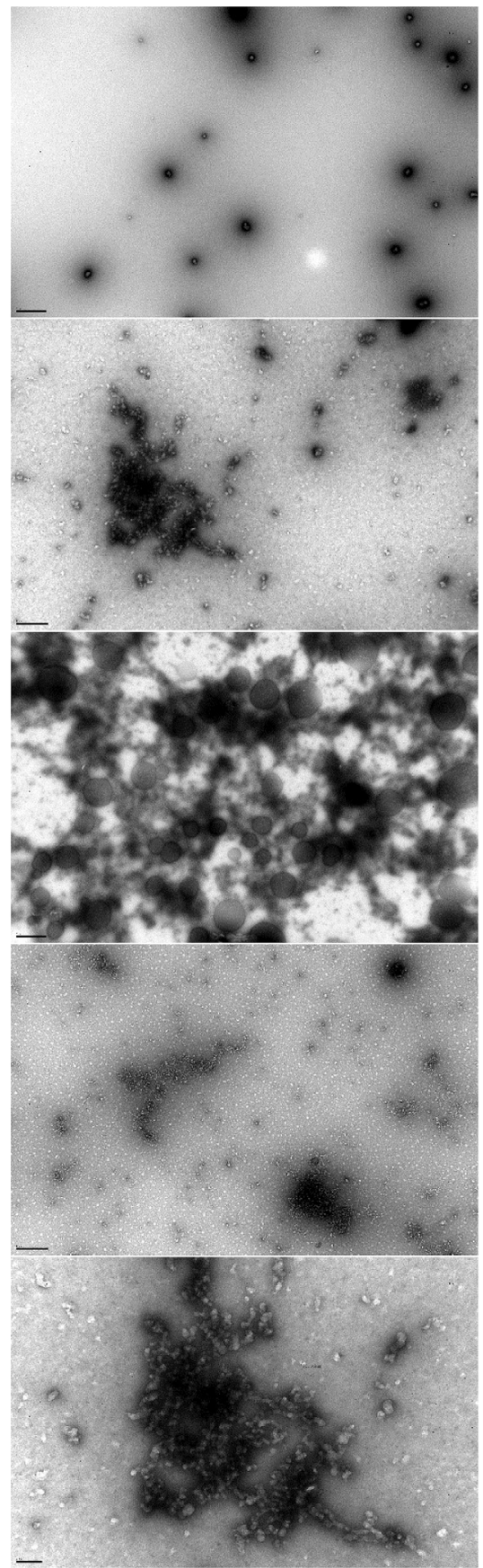

Fig. 3. - TEM micrographs of gastric and intestinal digestion of nanohydrogels with (A) and without (B) chitosan coating in the different compartments of a dynamic gastrointestinal system.

with micelle-like appearance. These micelles are called mixed micelles and are formed by bile salts and bile acids which are surfactants with an important role in the digestion of fats and fat-soluble nutrients (Verde \& Frenkel, 2010). The small particles also detected by size distributions can be explained by the degradation of the protein-based nanohydrogels. These results are in concordance with TEM images of the duodenum samples of Lf-GMP nanohydrogels. It is also possible to observe that nanohydrogels without chitosan coating are more heterogeneous, displaying particles with different sizes (Fig. 3B, in the duodenum compartment).

In the jejunal filtrate, it is possible to observe that the size of particles decreases and a second peak appears, indicating the presence of smaller particles. Fig. 3A, in the jejunal filtrate, also shows the presence of greater numbers smaller particles. In this step of digestion, samples pass through a hollow-fibre membrane, simulating the absorption that occurs in the small intestine where the larger particles are retained. In the ileum compartment, it is possible to observe the presence of large particles, due to aggregation of particles and enzymes, forming micelles. This is visible by the presence of two peaks with larger sizes (Fig. 2A, ileal delivery) and corroborated by TEM images (Fig. 3A, ileal delivery). In the ileal filtrate, all the small particles (Figs. $2 \mathrm{~A}$ and $3 \mathrm{~A}$ corresponding to the ileal filtrate) pass through the hollow-fibre membrane, again simulating the absorption in this part of the intestine. Fig. 2B shows that in the jejunal filtrate the particles with small sizes are present in higher numbers for nanohydrogels without chitosan coating than for nanohydrogels with chitosan coating at the same 
conditions (Fig. 2B in the jejunum compartment). This can be due to the presence of hydrolyzed proteins, indicating that nanohydrogels without chitosan coating more digested leading to the formation of smaller particles. A similar behaviour was observed for the ileal filtrate compartment, as can be seen in the corresponding graph and TEM micrograph of Figs. 2B and 3B.

In the ileal delivery, the size distribution shows two peaks of small particles with low intensity and one peak with particles with larger particles with high intensity (Fig. 2B, ileal delivery). This behaviour was already expected once the larger particles are delivered in this compartment. TEM images of ileal delivery (Fig. 3B) are in concordance with these results.

\subsection{In vitro digestion of a model lipophilic compound}

Nanohydrogels revealed a high ability to encapsulate and deliver lipophilic and hydrophilic bioactive compounds (Sanguansri and Augustin et al., 2006). However, their bioaccessibility after the passage by the gastrointestinal tract is unexplored, being extremely relevant to understand the behaviour of active compounds after ingestion.

Fig. 4 shows the amount of curcumin in the different compartments of the dynamic gastrointestinal system during in vitro digestion for free curcumin and curcumin encapsulated in coated nanohydrogels.

During gastric digestion, a decrease $(p<0.05)$ of free curcumin was observed while encapsulated curcumin did not show significant differences $(p>0.05)$. This can be explained by the fact that during gastric conditions, the $\mathrm{pH}$ of the stomach is around 2 and free curcumin has a very low aqueous solubility at acidic $\mathrm{pH}$ (Qian \& McClements, 2011; Yildirim, Oztop, \& Soyer, 2017). In nanohydrogels, curcumin is more stable and no phase separation was observed (results not shown).

In the duodenum an increase of curcumin solubility in the free form was observed, that can be due to the presence of digestion products such as mixed micelles that are capable to solubilize the lipophilic components (Pinheiro, Coimbra, \& Vicente, 2016). In the jejunal filtrate, it is possible to observe that the curcumin in free form is more available that in encapsulated form. This can be due to the fact, that at this stage the free curcumin has a lower size and a high solubility promoting a higher passage by a membrane.

During in vitro digestion, about $10.2 \pm 0.2 \%$ of the free curcumin was degraded whereas in the encapsulated form (in the nanohydrogel) this percentage was $8.1 \pm 0.1 \%$. Moreover, only $57.2 \pm 0.4 \%$ of the free curcumin was absorbed (amount of curcumin present in the jejunal and ileal filtrates) while $72 \pm 1.2 \%$ of encapsulated curcumin was absorbed. Chen, Li, and Tang (2015) observed similar behaviour for in vitro digestion of curcumin in soy protein isolate, indicating that the bioaccessibility of curcumin in the nanocomplexes was around $60 \%$, while in free form was of only $20 \%$ (Chen et al., 2015).

Fig. 5 shows the antioxidant activity of free curcumin and curcumin encapsulated in coated nanohydrogels.

$\mathrm{DPPH}$ is a free radical compound that has been used to evaluate the free-radical scavenging ability of samples. This method allows determining the anti-radical activity of an antioxidant by measuring the decrease in absorbance of DPPH radical caused by the scavenging of the hydroxyl radical concentration through hydrogen donation (Souza et al., 2012).

Fig. 5 shows that during gastric digestion, in the stomach compartment, a decrease of antioxidant activity of curcumin in both cases (curcumin in free form and curcumin encapsulated in nanohydrogels) was registered, when compared with the initial values. This can be related to the decrease of curcumin solubility in an acidic medium. During gastric digestion it was also observed that encapsulated curcumin showed a higher antioxidant activity $(p<0.05)$ when compared with curcumin in the free form, indicating that encapsulation protected its activity.

In duodenum, jejunum and ileum compartments a decrease of antioxidant activity of free curcumin was observed, which can be related to the hydrolytic degradation of curcumin in alkaline media (Walker, Gumus, Decker, \& McClements, 2017). When compared with encapsulated curcumin a significant decrease of antioxidant activity $(p>0.05)$ was observed, revealing that encapsulation is an effective means of maintaining the antioxidant activity of curcumin in alkaline solutions and digestion conditions.

Under simulated gastric and intestinal conditions, free curcumin lost around $68 \pm 0.1 \%$ of its antioxidant activity while when encapsulated in nanohydrogels only $30.2 \pm 0.1 \%$ of its activity was lost. These results are in agreement to the results reported by Blanco-Padilla, LópezRubio, Loarca-Piña, Gómez-Mascaraque, and Mendoza (2015) who observed that curcumin entrapped within fibres had a loss of antioxidant activity around $28 \%$ while free curcumin lost $72 \%$ during gastrointestinal digestion (Blanco-Padilla et al., 2015).

\subsection{In vitro digestion of a model hydrophilic compound}

Caffeine was used as a hydrophilic model compound, and its bioaccessibility as a free compound or encapsulated in coated nanohydrogels during gastrointestinal digestion was evaluated (Fig. 6).

During gastrointestinal digestion, it was observed that $18 \pm 1.2 \%$ of the encapsulated caffeine were degraded while in its free form the

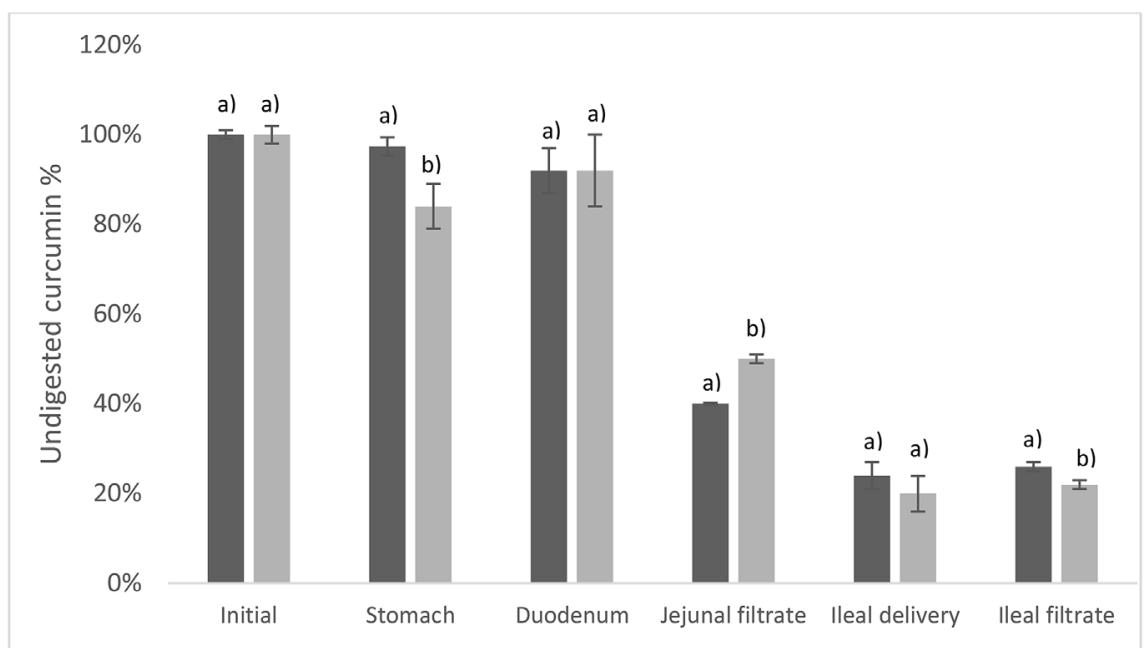

Fig. 4. - Undigested percentage of curcumin in the different compartments of the dynamic gastrointestinal system during in vitro digestion for free bioactive compound $(₫)$ and bioactive compound encapsulated in coated nanohydrogels ( $\square$ ) (data are presented as mean $\pm 95 \%$ confidence interval; values followed by different superscript letters are significantly different $(p<0.05))$. 


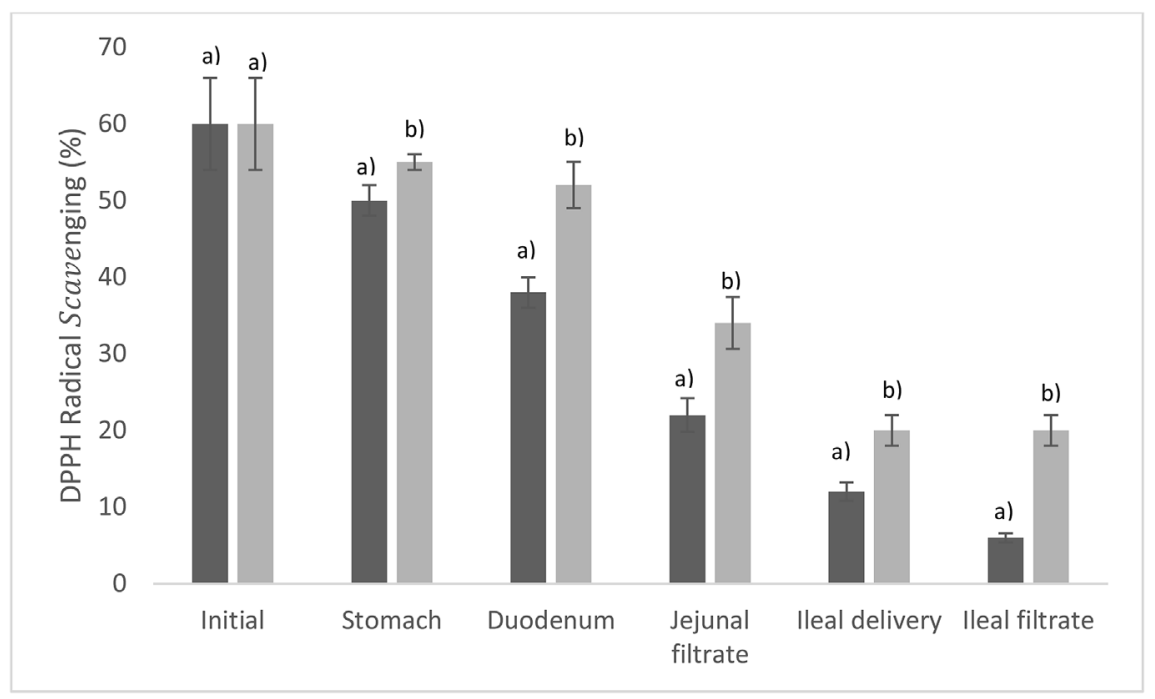

Fig. 5. - DPPH free radical scavenging activity of free curcumin ( $\square$ ) and curcumin encapsulated in nanohydrogels with chitosan coating $(\square)$ during gastrointestinal digestion (data are presented as mean $\pm 95 \%$ confidence interval; values followed by different superscript letters are significantly different $(p<0.05)$ ).

degradation was of $23 \pm 1.5 \%$. Moreover, the bioaccessibility of caffeine in coated nanohydrogels was $63.1 \pm 0.2 \%$ while in free form this value decreases to $59.2 \pm 2.1 \%$.

Stomach and duodenum solutions revealed that the amount of caffeine detected was not significantly different when comparing free and encapsulated forms. This is an indication that until this step of gastrointestinal digestion, caffeine stability, in both free and encapsulated forms, was not affected by the different gastrointestinal conditions to which it was submitted. However, in the jejunal filtrate solution, the amount of caffeine in free form was higher $(p<0.05)$ than that of encapsulated caffeine. The small size of caffeine and the high solubility of this bioactive compound in the gastrointestinal solutions promote its presence its passage to the filtrate more easily when in its free form. In the ileum compartment, a not significant difference $(p>0.05)$ between the amount of caffeine in free form and encapsulated form was observed. One of the major concerns of caffeine is the high absorption to human organism, and caffeine encapsulated can be a useful tool to promote a control release of this compound during gastro intestinal digestion. Guo, Harris, Kaur, Pastrana, \& Jauregi, 2017 observed that caffeine molecules were bound to the $\beta$-lactoglobulin after the gastro digestion indicating that the binding of caffeine to protein was not dependent on the microstructure but on the protein interaction (Guo et al., 2017).

\section{Conclusions}

A chitosan layer can be used to coat protein-based nanohydrogels and to improve the stability and the bioaccessibility of proteins and hydrophobic compounds eventually encapsulated in the nanohydrogels. This chitosan coating was able to decrease the degradation of lipophilic model compound (curcumin) during in vitro digestion and improve its bioaccessibility. In the case of a hydrophilic compound, it was observed that no significant differences were observed for bioaccessibility. These results have important implications for the design of effective proteinbased delivery systems for bioactive compounds.

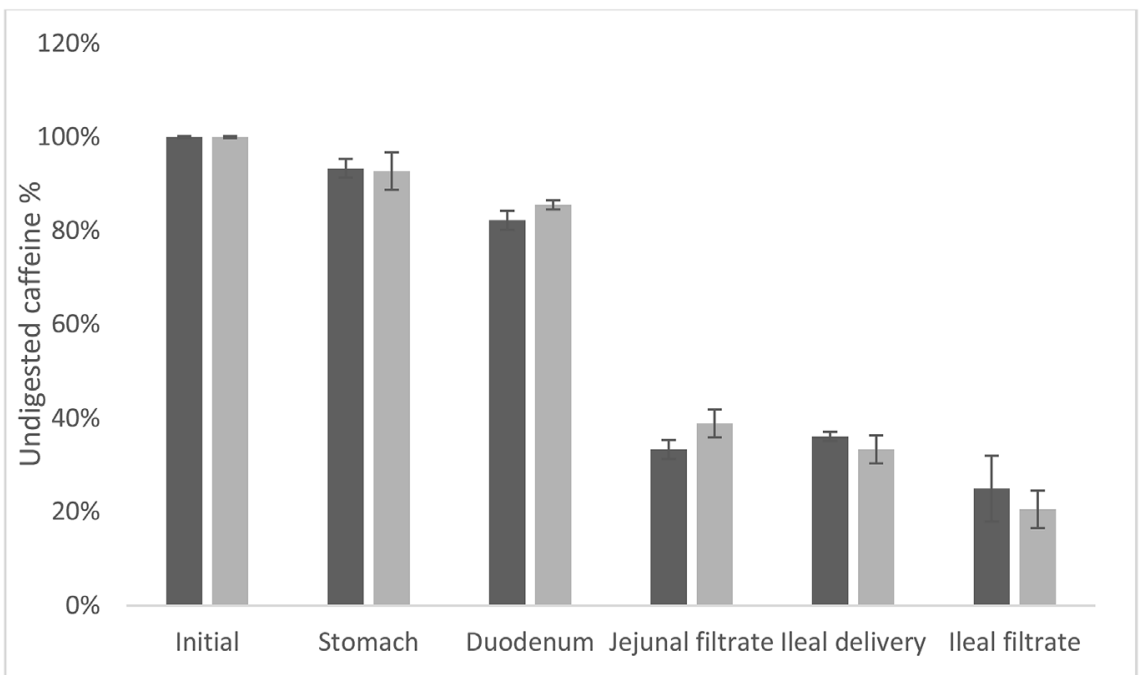

Fig. 6. Undigested percentage of caffeine in the different compartments of a dynamic gastrointestinal system during digestion of free caffeine $(\square)$ and caffeine encapsulated in coated nanohydrogels ( $\mathbf{\square}$ ) (data are presented as mean $\pm 95 \%$ confidence interval; values followed by different superscript letters are significantly different $(p<0.05)$ ). 


\section{Acknowledgements}

Ana I. Bourbon and Ana C. Pinheiro acknowledge the Fundação para a Ciência e Tecnologia (FCT, Portugal) for their fellowships SFRH/BD/ 73178/2010, and SFRH/BPD/101181/2014, respectively). The authors would like to acknowledge Rui Fernandes from IBMC, the University of Porto for assistance in taking the TEM pictures.

\section{Appendix A. Supplementary data}

Supplementary data related to this article can be found at http://dx. doi.org/10.1016/j.foodhyd.2018.06.015.

\section{References}

Ahmed, K., Li, Y., McClements, D. J., \& Xiao, H. (2012). Nanoemulsion- and emulsionbased delivery systems for curcumin: Encapsulation and release properties. Food Chemistry, 132(2), 799-807.

Anal, A. K., Bhopatkar, D., Tokura, S., Tamura, H., \& Stevens, W. F. (2003). ChitosanAlginate multilayer beads for gastric passage and controlled intestinal release of protein. Drug Development and Industrial Pharmacy, 29(6), 713-724.

Blanco-Padilla, A., López-Rubio, A., Loarca-Piña, G., Gómez-Mascaraque, L. G., \& Mendoza, S. (2015). Characterization, release and antioxidant activity of curcuminloaded amaranth-pullulan electrospun fibers. Lebensmittel-Wissenschaft und -Technologie- Food Science and Technology, 63(2), 1137-1144.

Blois, M. S. (1958). Antioxidant determinations by the use of a stable free radical. Nature, 181(4617), 1199-1200.

Bokkhim, H., Bansal, N., Grøndahl, L., \& Bhandari, B. (2016). In-vitro digestion of different forms of bovine lactoferrin encapsulated in alginate micro-gel particles. Food Hydrocolloids, 52, 231-242.

Bourbon, A. I., Pinheiro, A. C., Cerqueira, M. A., \& Vicente, A. A. (2016). Influence of chitosan coating on protein-based nanohydrogels properties and in vitro gastric digestibility. Food Hydrocolloids, 60, 109-118.

Chen, F.-P., Li, B.-S., \& Tang, C.-H. (2015). Nanocomplexation between curcumin and soy protein Isolate: Influence on curcumin stability/bioaccessibility and in vitro protein digestibility. Journal of Agricultural and Food Chemistry, 63(13), 3559-3569.

Guo, Y., Harris, P., Kaur, A., Pastrana, L., \& Jauregi, P. (2017). Characterisation of Blactoglobulin nanoparticles and their binding to caffeine. Food Hydrocolloids, 71, 85-93.

Inglingstad, R., Devold, T., Eriksen, E., Holm, H., Jacobsen, M., Liland, K., et al. (2010). Comparison of the digestion of caseins and whey proteins in equine, bovine, caprine and human milks by human gastrointestinal enzymes. Dairy Science \& Technology, 90(5), 549-563.

Liu, F., Gao, Y., \& Yuan, F. (2014). Effects of chitosan addition on in vitro digestibility of protein-coated lipid droplets. Journal of Dispersion Science and Technology, 36(11),
1556-1563.

Livney, Y. D. (2012). 10-Biopolymeric amphiphiles and their assemblies as functional food ingredients and nutraceutical delivery systems. In N. Garti, \& D. J. McClements (Eds.). Encapsulation Technologies and delivery systems for food ingredients and nutraceuticals (pp. 252-286). Woodhead Publishing.

Martins, J., Ramos, Ó., Pinheiro, A., Bourbon, A., Silva, H., Rivera, M., et al. (2015). Edible bio-based Nanostructures: Delivery, absorption and potential toxicity. Food Engineering Reviews, 1-23.

Nabil, S., Gauthier, S., Drouin, R., Poubelle, P., \& Pouliot, Y. (2011). In vitro digestion of proteins and growth factors in a bovine whey protein extract as determined using a computer-controlled dynamic gastrointestinal system (TIM-1). Food Digestion, 2(1-3), $13-22$.

Pinheiro, A. C., Coimbra, M. A., \& Vicente, A. A. (2016). In vitro behaviour of curcumin nanoemulsions stabilized by biopolymer emulsifiers - effect of interfacial composition. Food Hydrocolloids, 52, 460-467.

Qian, C., \& McClements, D. J. (2011). Formation of nanoemulsions stabilized by model food-grade emulsifiers using high-pressure homogenization: Factors affecting particle size. Food Hydrocolloids, 25(5), 1000-1008.

Rein, M. J., Renouf, M., Cruz-Hernandez, C., Actis-Goretta, L., Thakkar, S. K., \& da Silva Pinto, M. (2013). Bioavailability of bioactive food compounds: A challenging journey to bioefficacy. British Journal of Clinical Pharmacology, 75(3), 588-602.

Reis, P. M., Raab, T. W., Chuat, J. Y., Leser, M. E., Miller, R., Watzke, H. J., et al. (2008) Influence of surfactants on lipase fat digestion in a model gastro-intestinal system. Food Biophysics, 3(4), 370-381.

Sanguansri, P., \& Augustin, M. A. (2006). Nanoscale materials development - a food industry perspective. Trends in Food Science \& Technology, 17(10), 547-556.

Souza, B. W. S., Cerqueira, M. A., Bourbon, A. I., Pinheiro, A. C., Martins, J. T., Teixeira, J. A., et al. (2012). Chemical characterization and antioxidant activity of sulfated polysaccharide from the red seaweed Gracilaria birdiae. Food Hydrocolloids, 27(2), 287-292.

Su, D., \& Zhong, Q. (2016). Lemon oil nanoemulsions fabricated with sodium caseinate and Tween 20 using phase inversion temperature method. Journal of Food Engineering, $171,214-221$.

Troost, F. J., Steijns, J., Saris, W. H. M., \& Brummer, R.-J. M. (2001). Gastric digestion of bovine lactoferrin in vivo in adults. Journal of Nutrition, 131(8), 2101-2104.

Verde, A. V., \& Frenkel, D. (2010). Simulation study of micelle formation by bile salts. Soft Matter, 6(16), 3815-3825.

Walker, R. M., Gumus, C. E., Decker, E. A., \& McClements, D. J. (2017). Improvements in the formation and stability of fish oil-in-water nanoemulsions using Carrier oils: MCT, thyme oil, \& lemon oil. Journal of Food Engineering, 211, 60-68.

Wong, D. W. S., Camirand, W. M., Pavlath, A. E., Parris, N., \& Friedman, M. (1996) Structures and functionalities of milk proteins*. Critical Reviews in Food Science and Nutrition, 36(8), 807-844.

Yildirim, S. T., Oztop, M. H., \& Soyer, Y. (2017). Cinnamon oil nanoemulsions by spontaneous emulsification: Formulation, characterization and antimicrobial activity. Lebensmittel-Wissenschaft und -Technologie- Food Science and Technology, 84, 122-128.

Yvon, M., Beucher, S., Scanff, P., Thirouin, S., \& Pelissier, J. P. (1992). In vitro simulation of gastric digestion of milk proteins: Comparison between in vitro and in vivo data. Journal of Agricultural and Food Chemistry, 40(2), 239-244. 